\title{
Low-temperature infiltration identified using infrared thermography in patients with subcutaneous edema revealed ultrasonographically: A case report
}

\author{
Maiko Oya ${ }^{1}$, Toshiaki Takahashi ${ }^{1}$, Hidenori Tanabe ${ }^{2,3}$, Makoto Oe ${ }^{2}$, Ryoko Murayama ${ }^{2}$, \\ Koichi Yabunaka ${ }^{1}$, Yuko Matsui ${ }^{4}$, Hiromi Sanada ${ }^{1, *}$ \\ ${ }^{1}$ Department of Gerontological Nursing/Wound Care Management, Graduate School of Medicine, The University of Tokyo, Tokyo, \\ Japan; \\ ${ }^{2}$ Department of Advanced Nursing Technology, Graduate School of Medicine, The University of Tokyo, Tokyo, Japan; \\ ${ }^{3}$ Terumo Corporation, Tokyo, Japan; \\ ${ }^{4}$ School of Nursing, Kanazawa Medical University, Ishikawa, Japan.
}

\begin{abstract}
Summary Infiltration is a frequent complication of infusion therapy. We previously demonstrated the usefulness of infrared thermography as an objective method of detecting infiltration in healthy people. However, whether thermography can detect infiltration in clinical settings remains unknown. Therefore, we report two cases where thermography was useful in detecting infiltration at puncture sites. In both cases, tissue changes were verified ultrasonographically. The patients were a 56-year-old male with cholangitis and a 76-year-old female with hepatoma. In both cases, infiltration symptoms such as swelling and erythema occurred one day after the insertion of a peripheral intravenous catheter. Thermographic images from both patients revealed low-temperature areas spreading from the puncture sites; however, these changes were not observed in other patients. The temperature difference between the low-temperature areas and their surrounding skin surface exceeded $1.0^{\circ} \mathrm{C}$. Concurrently, ultrasound images revealed that tissues surrounding the vein had a cobblestone appearance, indicating edema. In both patients, subcutaneous tissue changes suggested infiltration and both had low-temperature areas spreading from the puncture sites. Thus, subcutaneous edema may indicate infusion leakage, resulting in a decrease in the temperature of the associated skin surface. These cases suggest that infrared thermography is an effective method of objectively and noninvasively detecting infiltration.
\end{abstract}

Keywords: Peripheral intravenous catheter, complications, early detection, ultrasonography

\section{Introduction}

Infiltration, one of the most frequent complications of infusion therapy with peripheral intravenous catheters (1), is defined as "inadvertent administration into the surrounding tissue" by the Infusion Nurses Society (INS) (2). When infusion agents leak from vessels and soak into the subcutaneous tissue, the contents, i.e., anticancer drugs and contrast media as well as other types of agents,

*Address correspondence to:

Hiromi Sanada, Department of Gerontological Nursing/ Wound Care Management, Graduate School of Medicine, The University of Tokyo, 7-3-1 Hongo, Bunkyo-ku, Tokyo 1130033, Japan.

E-mail: hsanada-tky@umin.ac.jp can cause erythema, induration, and even necrosis (3$5)$. Patients with infiltration often experience pain and in severe cases are left with persistent disabilities in their arms (6). Thus, infiltration can be a serious adverse event that warrants careful observation to detect abnormalities early during administration.

The INS have created an Infiltration Scale that is used to assess signs and symptoms such as "skin blanched", "edema", "cool to touch", "pain", and "numbness" (1). Even if patients are closely monitored for these signs and symptoms, confusion can often occur; as an example, agents can continue flowing despite obvious infiltration symptoms or can stop flowing without obvious infiltration symptoms. Hence, the assessment of infiltration can often be difficult in 
clinical settings.

Our group has sought to develop an objective method for early detection of infiltration by focusing on the symptom "cool to touch". Although infiltrating drugs are thought to lower the temperature of the surrounding tissues and the skin surface, few studies have examined this phenomenon. An experiment in dogs showed that microwave radiometry could be used to observe the decline in skin temperature during extravasation of doxorubicin into subcutaneous tissues (7). In addition, we used infrared thermography in an experiment to examine a model of infiltration in healthy adults (8). A circle-like low-temperature area around the puncture site was produced by intentionally allowing normal saline to leak into subcutaneous tissue; the resulting temperature differed from that obtained by the infusion of normal saline into the vessel. This thermal change occurred prior to pain manifesting, suggesting that thermography would be useful at early and noninvasive detection of infiltration. However, infiltration was intentionally produced in the model, so whether thermography could detect infiltration in patients with actual changes in their subcutaneous tissue was unknown.

Recently, ultrasonography has advanced greatly, particularly with respect to the depiction of peripheral veins. We previously reported that edema of the subcutaneous fat layer could be observed with ultrasound in patients with infiltration (9). To confirm whether thermographic images can practically indicate infiltration in patients with subcutaneous changes, we report two cases where thermal changes were evident in patients along with ultrasonographic signs and symptoms. Our contention is that the efficacy of thermography in detecting infiltration can be enhanced with ultrasonography.

\section{Case Report}

Adults undergoing peripheral intravenous catheterization in an internal medicine ward of a university hospital in Tokyo, Japan were monitored for infiltration. Infrared thermography and ultrasonography were performed on puncture sites immediately after catheter insertion and before removal. Ultrasonography was performed using portable ultrasound equipment (Noblus, Hitachi Aloka Medical Ltd., Tokyo, Japan) with a linear-array transducer (5-18.0 MHz). To identify the catheterized vein, the focus range and the image depth were set at 1.5$2.5 \mathrm{~cm}$. Infrared thermography images (Thermo Shot, Nippon Avionics Co. Ltd., Tokyo, Japan) were obtained with a range of $5.0^{\circ} \mathrm{C}$ and an emissivity value of 0.98 and those images were analyzed using the InfReC Analyzer NS9500 Lite (Nippon Avionics Co. Ltd., Tokyo, Japan). This study was approved by the Research Ethics Committee of the Graduate School of Medicine, the University of Tokyo (approval No. \#10348).
Figures 1 and 2 show the results for a patient who completed four days of infusion therapy without any complications. There were no macroscopic signs and symptoms, and none of the ultrasound images showed marked changes in the subcutaneous tissue, the vessel, or the catheter position upon insertion and removal. Both thermographic images showed similar temperature patterns around the puncture site. Two cases of patients with symptoms and signs of infiltration will now be reported.

\subsection{Case 1}

A 56-year-old man was admitted for cholangitis and a 22-gauge ethylene tetrafluoroethylene catheter (Surshield Surflo2, Terumo Corporation, Tokyo, Japan) was inserted in his right forearm to administer two doses of antibiotics and a continuous maintenance solution. A problem was noted at the puncture site, and the catheter removed approximately $23 \mathrm{~h}$ after insertion. Upon examination, swelling with a minor axis of $5 \mathrm{~cm}$ and a major axis of 6 $\mathrm{cm}$, cool skin, and paresthesia were noted at the puncture site, indicating Grade 2 infiltration according to the Infiltration Scale.

Ultrasound images taken before the removal of the catheter revealed subcutaneous edema as indicated by tissue with a cobblestone appearance that was not present at the time of insertion; however, the catheter was still in the vessel (Figure 3). Thermographic images showed a low-temperature area mainly at the dressing site at the time of insertion (Figure 4A), but a low-temperature area spreading from the puncture site was observed when the catheter was removed (Figure 4B). This low-temperature area corresponded to the site of the swelling. The temperature at the center of the low-temperature area was $32.2^{\circ} \mathrm{C}$ and that in the surrounding area was $33.4^{\circ} \mathrm{C}$, indicating a temperature difference of $1.2^{\circ} \mathrm{C}$.

\subsection{Case 2}

A 76-year-old woman was admitted for radiofrequency ablation of a hepatoma. A 22-gauge catheter of the same type used in Case 1 was inserted in her left forearm and retained with a heparin lock. During the procedure, glucose and Ringer's lactate solutions were administered together with antibiotics and an analgesic. However, a problem at the puncture site was noted approximately 25 $\mathrm{h}$ after catheter insertion while the patient was receiving postoperative glucose and Ringer's lactate infusions. Examination revealed swelling and erythema with a minor axis of $2 \mathrm{~cm}$ and a major axis of $3 \mathrm{~cm}$, pain on movement, and cool skin, indicating Grade 2 infiltration according to the Infiltration Scale.

Ultrasound images before catheter removal showed tissue with a cobblestone appearance and thicker subcutaneous tissue than was noted at the time of insertion (Figure 5). Although the vein was barely visible 

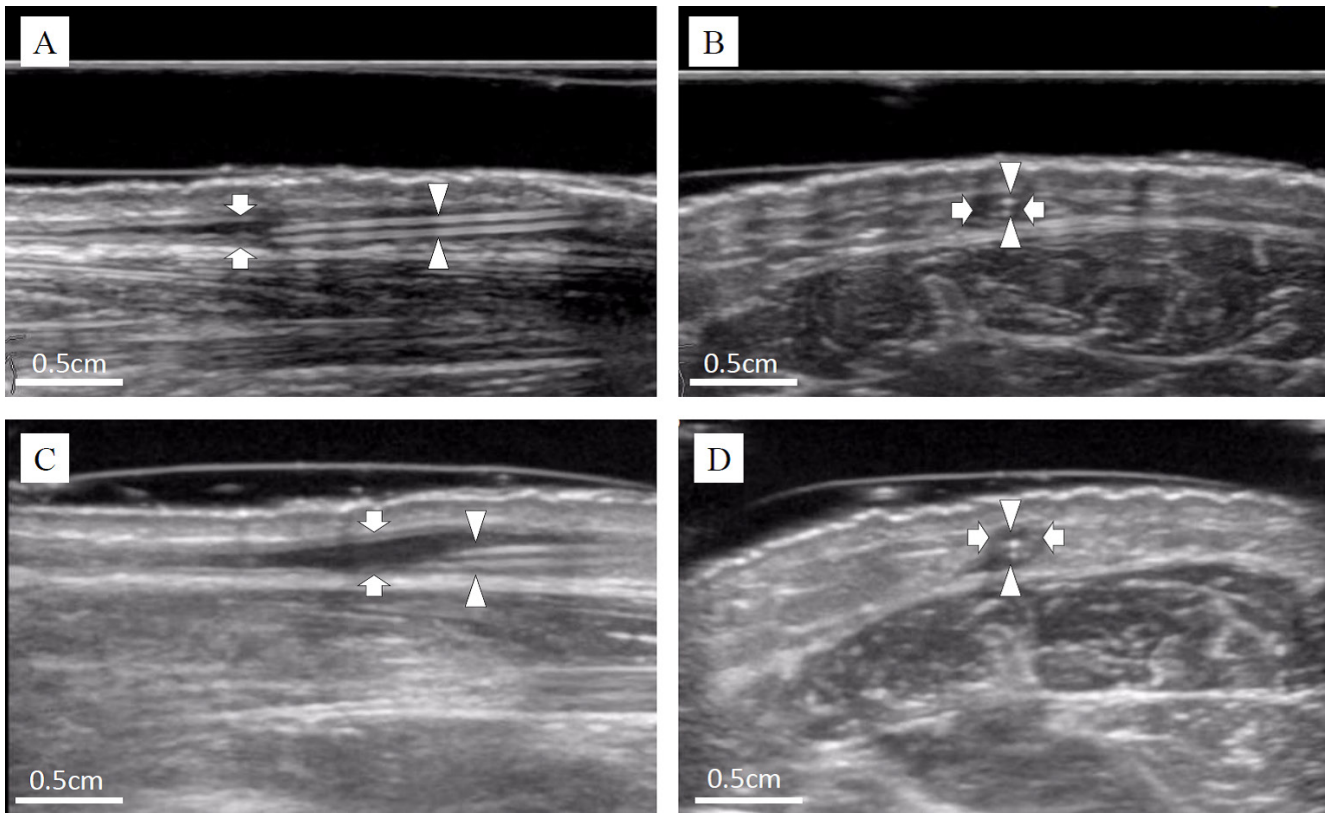

Figure 1. Representative ultrasound images from a normal patient. (A) Longitudinal and (B) transverse scan after catheter insertion; (C) longitudinal and (D) transverse scan before removal (4 days after insertion). Arrowheads indicate the catheter, with the vein shown between arrows. There were no marked differences between the time of insertion and removal.
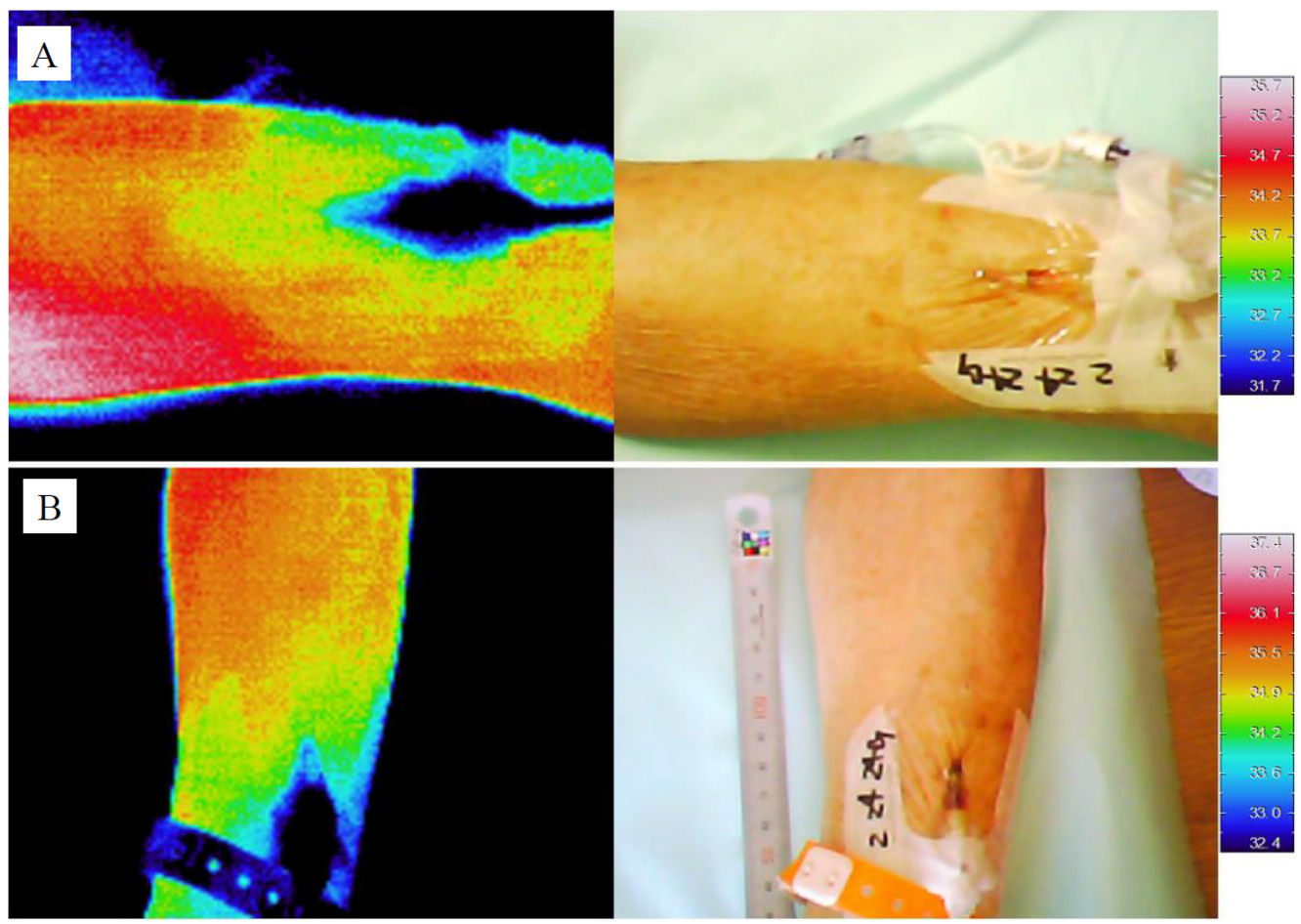

Figure 2. Representative thermographic and macroscopic images from a normal patient. (A) After insertion and (B) before removal. There were no complications at the time of removal, and similar temperature patterns were observed.

because of its small diameter, the catheter appeared to be within the vessel. Figure $6 \mathrm{~B}$ shows a thermographic image with a widely spread low-temperature area at the time of removal, whereas figure 6A shows no lowtemperature area at the time of insertion. The temperature was $31.9^{\circ} \mathrm{C}$ at the center of the low-temperature area and $33.3^{\circ} \mathrm{C}$ outside that area, indicating a temperature difference of $1.4^{\circ} \mathrm{C}$.

\section{Discussion}

Both of the current patients displayed infiltration symptoms during peripheral intravenous catheterization. Infrared thermography revealed a low-temperature area in both patients, and the temperature of this area differed by more than $1.0^{\circ} \mathrm{C}$ in comparison to the temperature of the surrounding tissue. Ultrasonographic changes in 

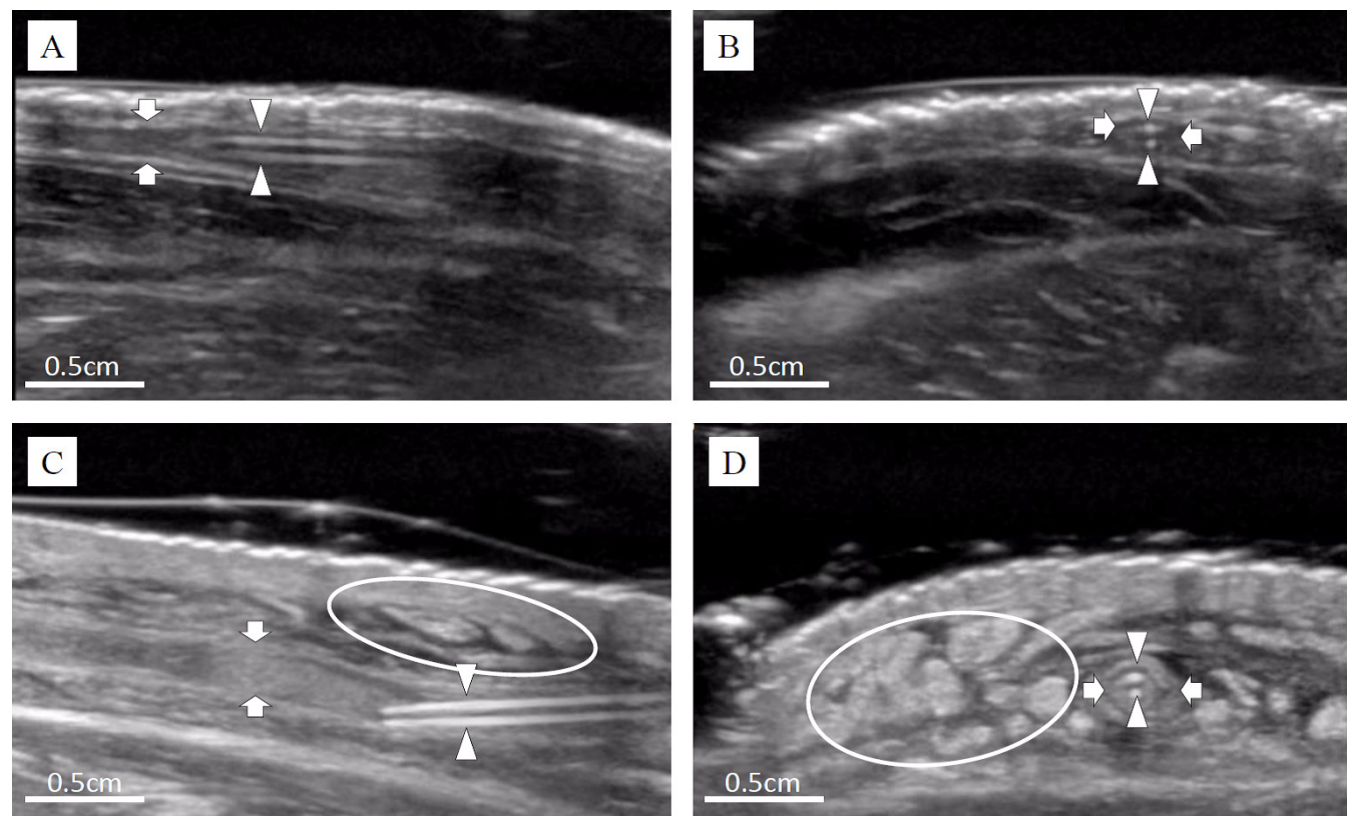

Figure 3. Ultrasound images from the patient in Case 1. Images (A) and (B) are after insertion and images (C) and (D) are before removal, when infiltration symptoms were observed. Arrowheads indicate the catheter, and the vein is between the arrows. Images (C) and (D) show tissue with a cobblestone appearance around the vein (circled area).

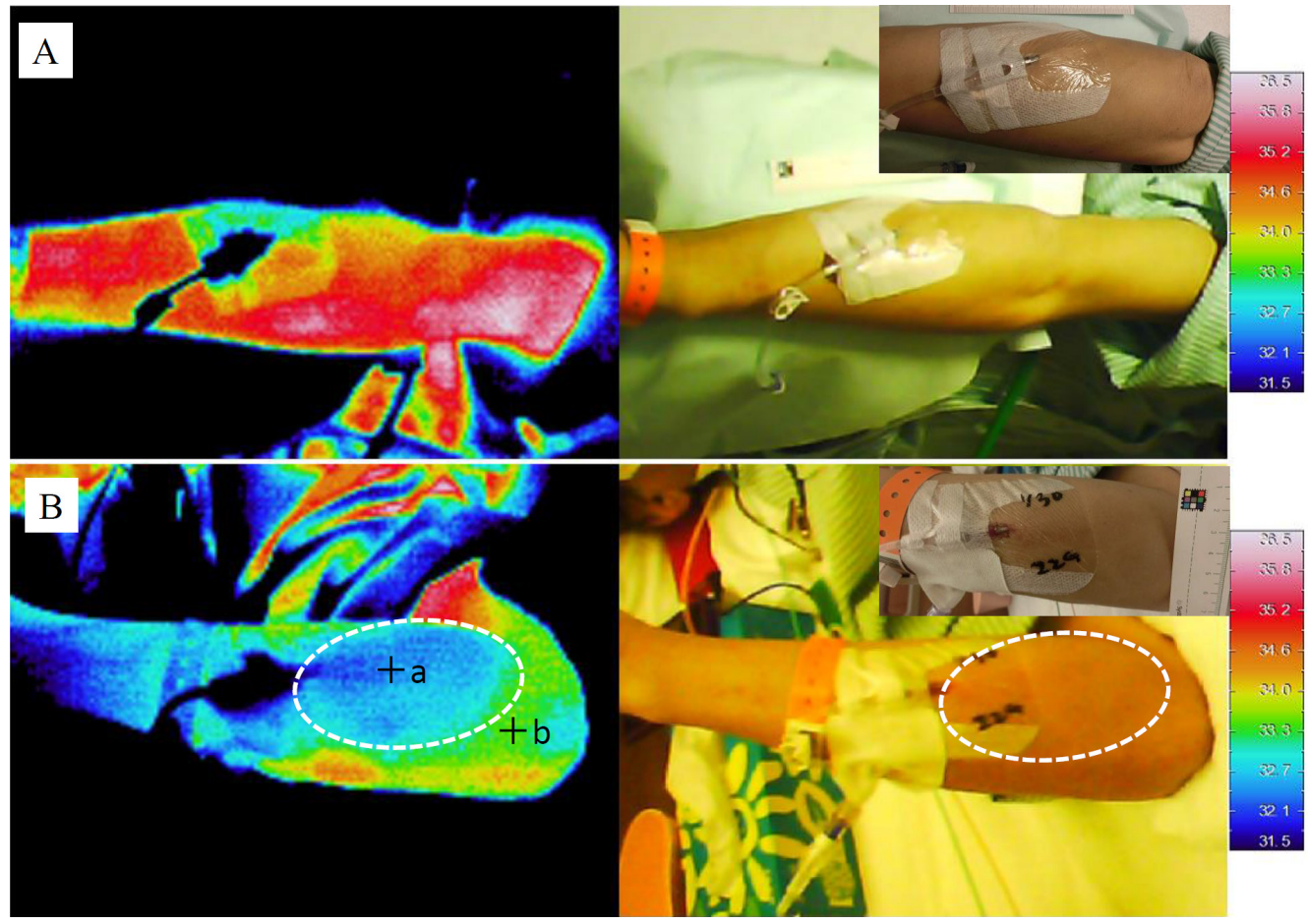

Figure 4. Thermographic and macroscopic images from the patient in Case 1. (A) After insertion; (B) before removal (with clear visible images). Image (B) shows a low-temperature area spreading from the puncture site. The center of the lowtemperature area (a) was $32.2^{\circ} \mathrm{C}$ and the surrounding area (b) was $33.4^{\circ} \mathrm{C}$.

subcutaneous tissue were evident in both patients, and these changes (e.g. tissue with a cobblestone appearance) were indicative of edema. The drop in the temperature of the tissue and skin surface may have been the result of an infusion leaking into surrounding tissues. Thermographic observation of the puncture site was therefore a useful method of detecting subcutaneous abnormalities during infusion in these patients.

An experiment involving extravasation of doxorubicin in dogs showed that a temperature decrease of more than $1.0^{\circ} \mathrm{C}$ was likely to indicate infiltration (7). This finding is supported by evidence that skin temperature decreases less than $1.0^{\circ} \mathrm{C}$ during proper intravenous therapy. In both of the current patients, the 

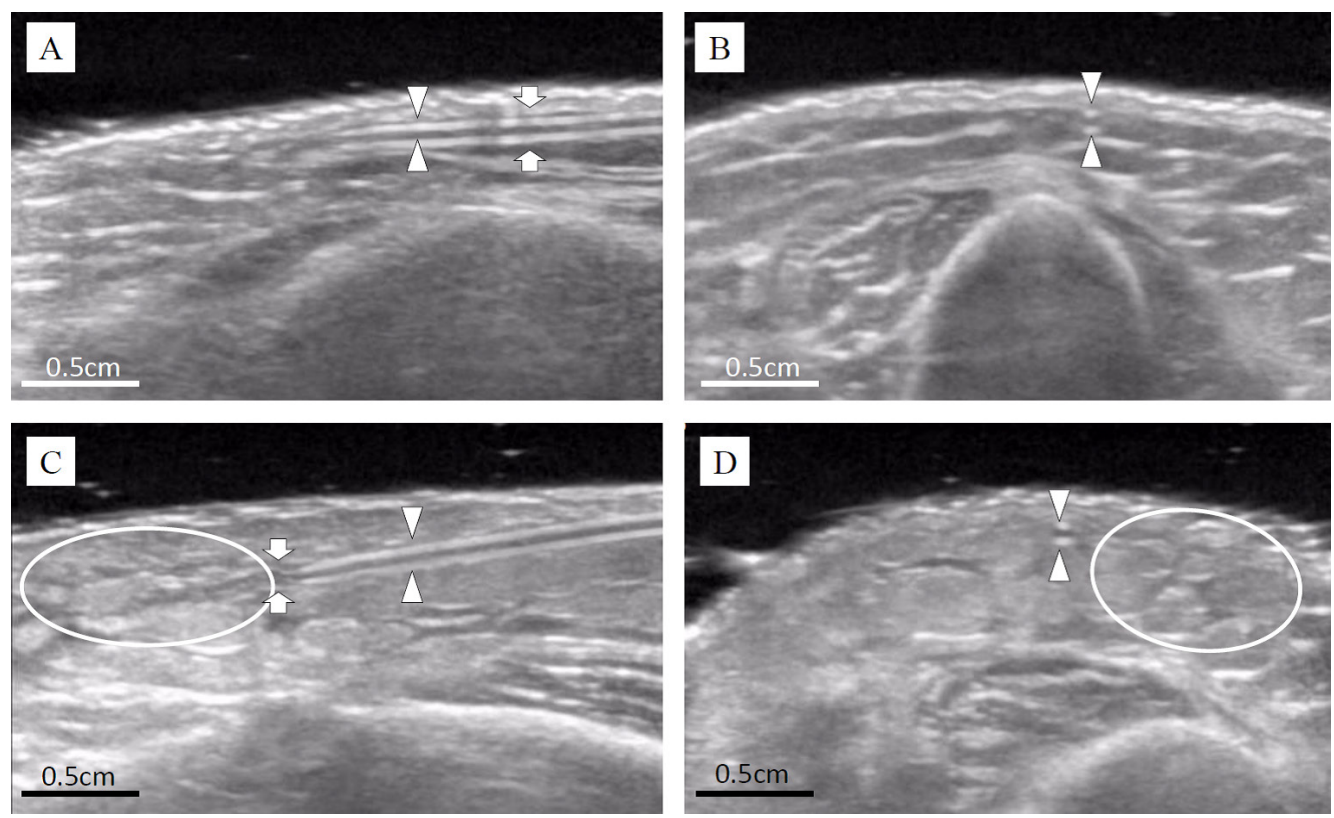

Figure 5. Ultrasound images from the patient in Case 2. Images (A) and (B) are after insertion and images (C) and (D) are before removal, when infiltration symptoms were observed. Arrowheads indicate the catheter, and the vein is between the arrows. Images (C) and (D) show surrounding tissue with a cobblestone appearance.

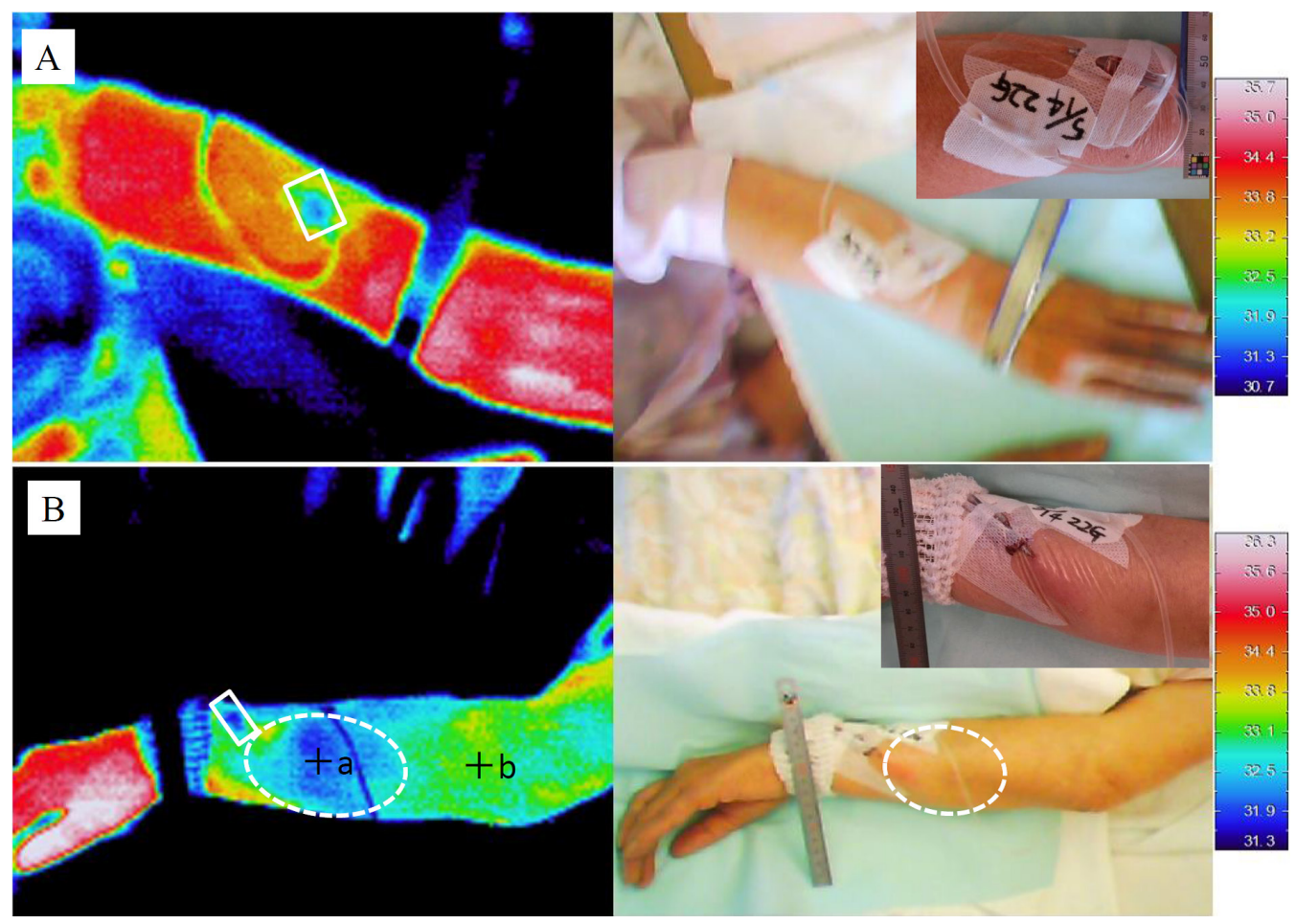

Figure 6. Thermographic and macroscopic images from the patient in Case 2. (A) After insertion and (B) before removal (with clear visible images). The rectangle represents the position of the catheter hub. Image (B) shows a low-temperature area spreading widely from the puncture site (circled area). The center of the low-temperature area (a) was $31.9^{\circ} \mathrm{C}$, and the area beyond the lowtemperature area (b) was $33.3^{\circ} \mathrm{C}$.

temperature decrease in comparison to surrounding areas was greater than $1.0^{\circ} \mathrm{C}$, suggesting a potential threshold for the detection of abnormalities during peripheral intravenous catheterization. However, the experiment in dogs showed that greater amounts of extravasation and more rapid flow rates caused a sharper drop in temperature. The thermography images in this case report were taken after infusions were stopped, but observations at different times and different rates of infusion could result in substantial disparities in the temperature difference.

In a previous study, we examined a model of infiltration by inserting a catheter directly into subcutaneous tissue, producing a low-temperature area 
that appeared as a localized circle at the tip of the catheter on thermography (8). In both of the current cases, the low-temperature area spread from the puncture site but ultrasound images showed no deviation of the catheter from the vessel. The actual phenomena of infiltration may produce different thermal patterns on the skin surface depending on where a catheter is inserted. Further research with a larger sample is needed to identify patterns of temperature changes, but the current cases do offer clues to diagnostic information to help with the objective and noninvasive diagnosis of infiltration.

Several techniques have been reported to decrease complications in infusion therapy $(10,11)$. However, evaluating macroscopic symptoms objectively is difficult. For example, patients who are unconscious or who have peripheral neuropathy cannot feel or complain of pain. In such instances, thermographic evaluation could provide an objective and reliable method of detecting infiltration.

In both of the current patients with infiltration, there was evidence of a relationship between a spreading lowtemperature area and subcutaneous edema depicted ultrasonographically. However, further research is needed to determine when and how to judge abnormalities based on thermal changes during peripheral intravenous catheterization. Those findings could allow infrared thermography to be used to detect infiltration.

\section{Acknowledgements}

This work was conducted as a part of a project funded by the JSPS KAKENHI Grant Number 26670915.

\section{References}

1. Rickard CM, Webster J, Wallis MC, Marsh N, McGrail MR, French V, Foster L, Gallagher P, Gowardman JR, Zhang L, McClymont A, Whitby M. Routine versus clinically indicated replacement of peripheral intravenous catheters: A randomised controlled equivalence trial. Lancet. 2012; 380:1066-1074.

2. Infusion Nurses Society. Infusion Nursing Standards of Practice. J Infus Nurs. 2006; 29:S1-S92.

3. European Oncology Nursing Society. Extravasation guidelines 2007. http://www.cancernurse.eu/documents/ EONSClinicalGuidelinesSection6-en.pdf (accessed September 30, 2015).

4. Wang CL, Cohan RH, Ellis JH, Adusumilli S, Dunnick NR. Frequency, management, and outcome of extravasation of nonionic iodinated contrast medium in 69,657 intravenous injections. Radiology. 2007; 243:8087.

5. Dychter SS, Gold DA, Carson D, Haller M. Intravenous therapy: A review of complications and economic considerations of peripheral access. J Infus Nurs. 2012; 35:84-91.

6. Hadaway L. Infiltration and extravasation. Am J Nurs. 2007; 107:64-72.

7. Shaeffer J, el-Mahdi AM, Hamwey AE Jr, Carr KL. Detection of extravasation of antineoplastic drugs by microwave radiometry. Cancer Lett. 1986; 31:285-291.

8. Matsui Y, Murayama R, Tanabe H, Oe M, Fukuda M, Motoo Y, Wagatsuma T, Kinoshita S, Sakai K, Konya C, Sugama J, Sanada H. Analysis of thermographic images in an extravasation model of intravenous infusion in healthy adults. Journal of Nursing Science and Engineering. 2014; 1:4-11. (in Japanese)

9. Yabunaka K, Murayama R, Takahashi T, Tanabe H, Kawamoto A, Oe M, Arai R, Sanada H. Ultrasonographic appearance of infusion via the peripheral intravenous catheters. Journal of Nursing Science and Engineering. 2015; 2:40-46. (in Japanese)

10. Doellman D, Hadaway L, Bowe-Geddes LA, Franklin M, LeDonne J, Papke-O'Donnell L, Schulmeister L, Stranz M. Infiltration and extravasation: Update on prevention and management. J Infus Nurs. 2009; 32:203-211.

11. Schulmeister L. Extravasation management: Clinical update. Semin Oncol Nurs. 2011; 27:82-90.

(Received April 12, 2016; Accepted April 16, 2016) 